\title{
$\alpha$ 螺旋构象对模型寡肽与聚合物相互作用的影响
}

\author{
李纪红 ${ }^{\oplus \otimes}$ 党琴琴 ${ }^{\circledR}$ 孙宏伟 ${ }^{\circledR}$ 查瑞涛 $^{\circledR}$ 袁 直 $^{{ }^{*}}$
}

(1) 南开大学化学学院, 天津 300071; (2) 天津医科大学基础医学院, 天津 300070; (3) 天津科技大学材料科学与化学工程学院, 天津 300457.*联系人, E-mali: zhiy@nankai.edu.cn)

摘要 通过核磁技术中的化学位移微扰法和分子模拟技术，在分子水平上揭示了二甲基胺修饰聚丙烯 酰胺吸附剂(DMAPAM)对八肽 VVRGCTWW(V8)和四肽 CTWW(C4)吸附能力差异的原因. 结果表明, 除了非键的相互作用影响吸附剂对多肽的吸附能力外, 寡肽的构象是造成对多肽吸附能力差异的重要 因素. V8 的 $\alpha$ 螺旋结构使得 V8 与吸附剂间可以形成多种弱相互作用力协同作用的模式，从而显著提高 吸附剂对其的吸附能力. 该结果对了解生物材料与多肽间的作用机理具有重要意义.

\section{关键词 寡肽 构象 聚合物 作用机制}

近年来，随着合成高分子在生物材料领域应用 的日趋广泛, 多肽与高分子聚合物的相互作用机理 成为人们关注的热点. 研究主要涉及多肽尤其是寡 肽与聚合物作用时，多肽的一级序列对相互作用的

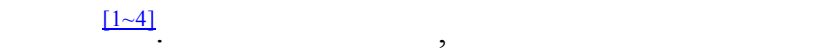
发生相互作用后, 构象会改变而形成有序的二级结 构 ${ }^{[5,6]}$, 但未见关于多肽二级结构对其与聚合物相互 作用影响的研究报道. 本文在研究吸附剂对寡肽吸 附时发现, 寡肽的二级结构对其与聚合物的相互作 用具有非常重要的影响.

$\mathrm{V} 8$ 是一种从尿毒症患者血液中分离出的中分子 多肽, 其一级序列为 VVRGCTWW ${ }^{[7]}$. 为清除该物质, 我们通过Mannichi 反应，合成了一种改性的二甲基 胺修饰聚丙烯酰胺吸附剂(DMAPAM) ${ }^{[8]}$. 前期研究 表明, DMAPAM与V8 的主要作用位点位于C端的Trp. 为了考察多肽二级结构对吸附剂吸附能力的影响, 人工合成了臭肽 $\mathrm{C} 4$, 其一级序列为 CTWW. C4 中含 有DMAPAM与V8 的作用位点 Trp, 且 Trp的位置及主 要相邻氨基酸残基在两条肽中完全一样，由此可以 排除邻位效应的影响; 此外, 两条肽中所含疏水和亲 水氨基酸的比例相同，肽的亲疏水性质不会受到明 显影响.

为了考察 DMAPAM 对两个葟肽的吸附情况，进 行了静态吸附实验. 取 $3 \mathrm{~mL}$ 预先配制好的多肽水溶 液, 注入 $10 \mathrm{~mL}$ 比色管中, 加入 $0.2 \mathrm{~g}$ 充分溶胀后的 吸附剂 DMAPAM, 加塞密封后置于摇床中匀速摇动, $37^{\circ} \mathrm{C}$ 下进行吸附. 每隔 $10 \mathrm{~min}$ 定时取样, 通过吸光度
的变化计算吸附剂对多肽的吸附量, 直至吸附达到 动态平衡. 结果如表 1 所示, 尽管两条肽的结构相似, 但是吸附剂 DMAPAM 对 $\mathrm{C} 4$ 的吸附能力极低, 在 $\mathrm{pH}$ 值为 5.4 时, 吸附量甚至为 0 .

表 1 吸附剂 DMAPAM 对多肽的吸附结果

\begin{tabular}{cccc}
\hline \multirow{2}{*}{ 多肽 } & \multicolumn{3}{c}{ 吸附量 $/ \mathrm{mg} \cdot \mathrm{g}^{-1}$ (湿球) } \\
\cline { 2 - 4 } & $\mathrm{pH}=4$ & $\mathrm{pH}=5.4$ & $\mathrm{pH}=9$ \\
\hline $\mathrm{V} 8$ & 0.318 & 0.541 & 0.598 \\
$\mathrm{C} 4$ & 0.024 & -0 & 0.045 \\
\hline
\end{tabular}

核磁技术中的化学位移微扰法是测定分子间相 互作用位点的有力手段, 但是该方法仅适用于液相 体系. 因此，我们合成了水溶性线型的 DMAPAM, 采集了 $\mathrm{C} 4$ 与 DMAPAM 作用前后的同核化学位移 全相关谱(TOCSY), 如图 1 所示.

C4 是含 4 个氨基酸残基的寡肽, 链段具有非常 好的柔顺性, C4 中任一个氨基酸残基都有可能与聚 合物发生相互作用. 图 1 结果显示, 除了碳端的 Trp 残基中 $\alpha \mathrm{N}$ 上的质子向高场移动了 -0.02 , 其他氨基酸 残基的化学位移都没有受到聚合物的影响. 这表明 DMAPAM 与 C4 的相互作用位点也在碳端 Trp.

吸附结果显示, 体系 $\mathrm{pH}$ 值对 DMAPAM 吸附 V8 具有显著影响, 由此看来对多肽吸附能力的差异有 可能是电荷性质的差异造成的. 利用 $\zeta$ 电势滴定法, 测定了 DMAPAM 在不同 $\mathrm{pH}$ 下的带电情况, 结果如 图 2 所示. 由滴定曲线得到 DMAPAM 的等电点是 8.33. 同时, 利用 DNAMAN 软件计算了 $\mathrm{V} 8$ 和 $\mathrm{C} 4$ 的 等电点, 分别为 8.66 和 5.22 . 


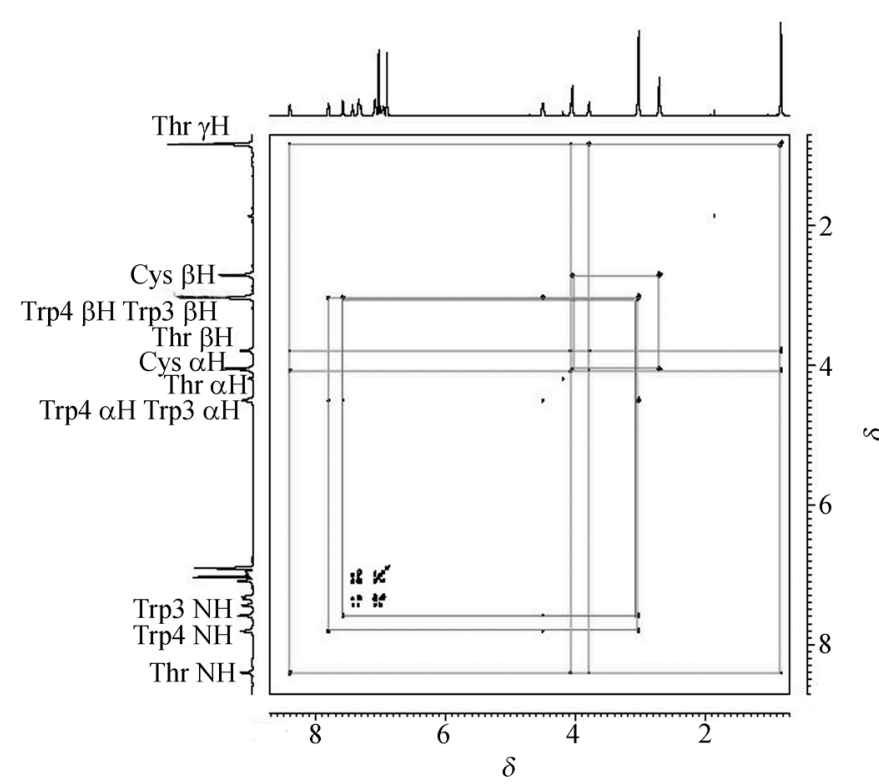

(a)

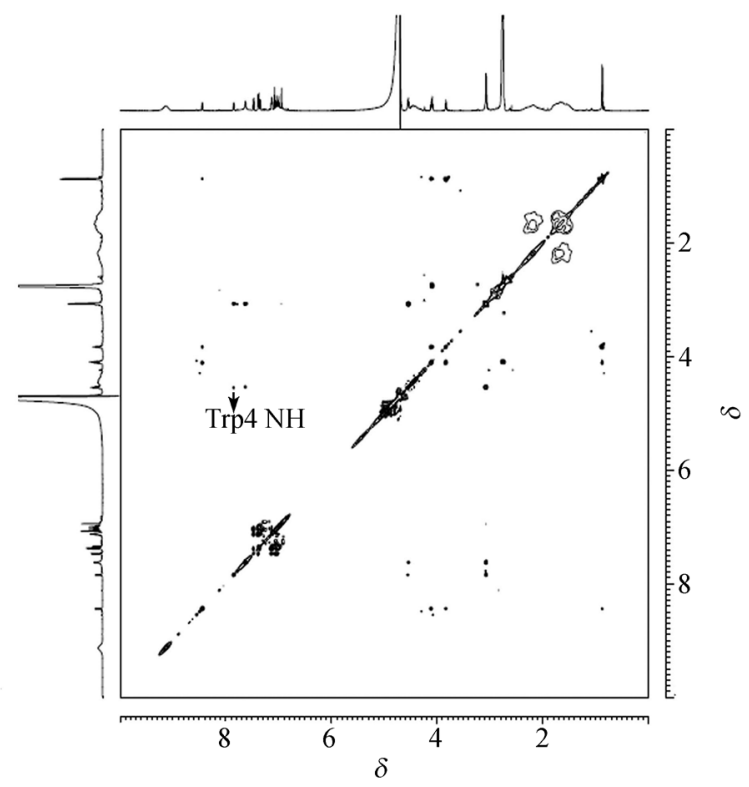

(b)

图 $1 \mathrm{C} 4$ 与聚合物 DMAPAM 相互作用的 TOCSY 谱图

(a) $\mathrm{C} 4\left(0.5 \mathrm{~mL} 10 \mathrm{mmol} / \mathrm{L}\right.$ 含 $10 \% \mathrm{D}_{2} \mathrm{O}$ 水溶液)的 TOCSY 谱及谱图归属; (b) C4 与 DMAPAM 等物质量的混合样品 $(0.5 \mathrm{~mL} 10 \mathrm{mmol} / \mathrm{L}$ 含 $10 \% \mathrm{D}_{2} \mathrm{O}$ 水溶液)的 TOCSY 谱

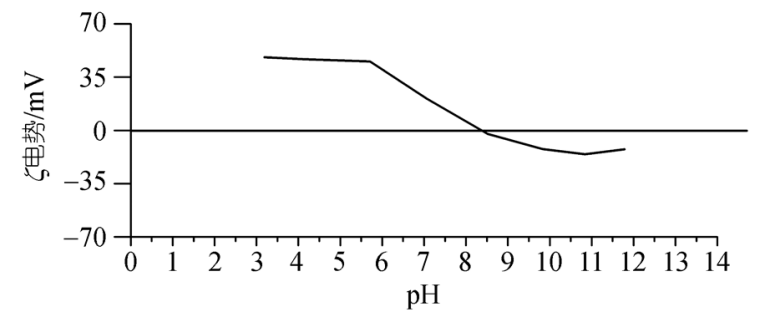

图 2 聚合物 DMAPAM 的 $\zeta$ 电势-pH 滴定曲线

由等电点的测定结果可知, 当溶液的 $\mathrm{pH}$ 为 5.4 时 DMAPAM带正电, 而V8此时带正电, C4 应该带负电. 根据“同性相斥, 异性相吸”的原理, DMAPAM对C4 吸附能力应强于V8, 但吸附试验结果正好与之相悖. 这一结果表明, DMAPAM与V8 相互作用的主要驱动 力不只是静电力. Tribet等人 [9]曾经报道由于疏水作 用的驱动, 疏水改性的聚阴离子可以与带负电的牛 血清蛋白 BSA形成复合物. 由此我们认为, 在 DMAPAM与V8 间还存在静电作用之外的弱相互作 用力, 从而弥补静电斥力的影响.

在前期的研究中，核磁及园二色分析结果均证 明寡肽V8 具有 $\alpha$ 螺旋结构 ${ }^{[7]}$. 为了考察多肽二级构象 对吸附能力的影响, 我们分别用Sybyl6.91 建立了 $\alpha$ 螺 旋的 V8, 无规的 V8 和无规 $\mathrm{C} 4$ 的分子模型, 用 Autodock3.0 软件对吸附剂模型和多肽模型进行了分
子对接 ${ }^{[10,11]}$. 对接构象最低能量结果见表 2. 显然, 无规构象的 C4 和V8 与DMAPAM的结合能明显低于 $\alpha$ 螺旋构象的 V8. 对接的最低能量构象模型直观地 给出了造成这一结果的原因(图 3). 图3(a)显示具有 $\alpha$ 螺旋的V8 不仅与DMAPAM对接的位点与核磁结果 完全吻合, 而且由于V8 的 $\alpha$ 螺旋结构, C端两个 Trp 残基可以形成一个疏水的钳式结构，有利于 DMAPAM的配体插入, 形成疏水、氢键和静电的多 重协同作用. 相对而言, 不具有 $\alpha$ 螺旋的寡肽则没有这 样的钳式结构(图 3(b)和(c)). 虽然四肽与DMAPAM间 能形成氢键作用, 但是暴露在水体系中, 氢键会被大 大削弱, 因此不存在弱相互作用的加合效果, 所以作 用力明显减弱.

表 2 分子对接最低能量构象的结合能

\begin{tabular}{lccc}
\hline & $\alpha$ 螺旋构象 V8 & 无规构象 V8 & 无规构象 C4 \\
\hline 结合能 $/ \mathrm{kJ} \cdot \mathrm{mol}^{-1}$ & -37.97 & -28.73 & -27.05 \\
\hline
\end{tabular}

核磁实验中给出在 DMAPAM 与 $\mathrm{C} 4$ 间可能存在 静电引力, 但静态吸附实验中 DMAPAM 对 C4 几乎 没有吸附的实验结果, 可能是由于 DMAPAM 和 $\mathrm{C} 4$ 之间只存在单点的静电相互作用, 吸附速率和解吸 速率基本相等, 并且是一个快速过程, 所以表观上没 有明显的吸附。 


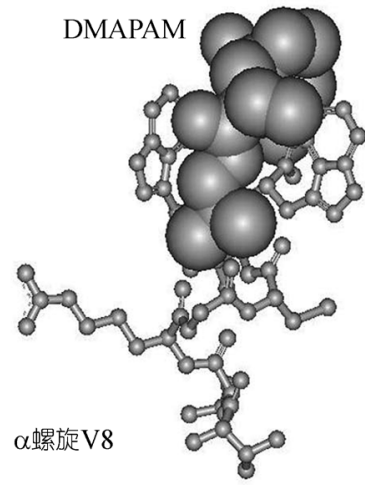

(a)

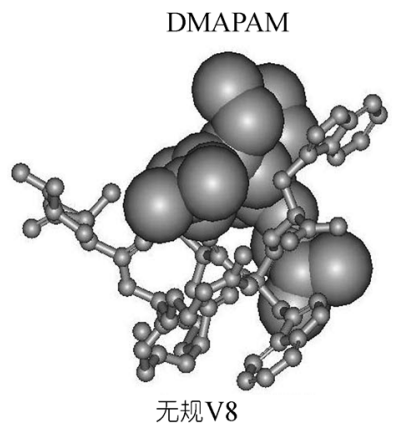

(b)

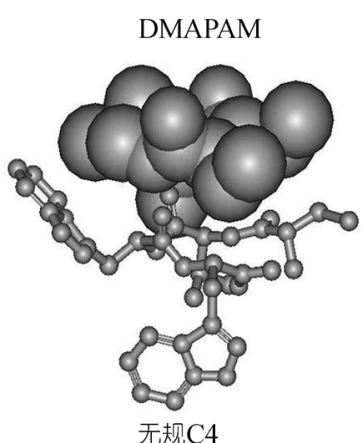

(c)

图 3 不同构象多肽与 DMAPAM 分子对接最低能量构象模型

综上所述，由于 $\mathrm{V} 8$ 的 $\alpha$ 螺旋结构存在一个疏水 的钳式区域，能够形成多种弱相互作用的协同效应， 这是造成 DMAPAM 对 V8 和 C4 吸附能力差别的关 键因素. 这一结论揭示了寡肽的二级结构在多肽与 聚合物的相互作用中的重要作用，对了解生物材料 与多肽间的作用机理具有重要意义.

\section{参考文献}

1 Jódar-Reyes A B, Ortega-Vinuesa J L, Martín-Rodríguez A. Adsorption of different amphiphilic molecules onto polystyrene lattices. J Colloid Interface Sci, 2005, 282(2): 439-447[DOI]

2 Cárdenas M, Schillén K, Pebalk D, et al. Interaction between DNA and charged colloids could be hydrophobically driven. Biomacromolecules, 2005, 6: 832-837[DOI]

3 Huang H M , Chen W Y, Ruaan R C. Microcalorimetric studies of the mechanism of interaction between designed peptides and hydrophobic adsorbents. J Colloid Interface Sci, 2003, 263(1): 23-28[DOI]

4 Renner C, Piehler J, Schrader T. Arginine- and lysine-specific polymers for protein recognition and immobilization. J Am Chem Soc, 2006, 128(2): 620-628[DOI]
5 Verma A, Nakade H, Simard J M, et al. Recognition and stabilization of peptide $\alpha$-helices using templatable nanoparticle receptors. J Am Chem Soc, 2004, 126(35): 10806-10807[DOI]

6 Tashiro S, Tominaga M, Yamaguchi Y, et al. Peptide recognition: Encapsulation and a-helical folding of a nine-residue peptide within a hydrophobic dimeric capsule of a bowl-shaped host. Chem Eur J, 2006, 12(12): 3211-3217[DOI]

7 李国华, 李纪红, 杨眉, 等. 尿毒症毒素多肽二级结构的测定与 预测. 高等学校化学学报, 2005, 26(10): 1855-1857

8 Li G H, Li J H, Wang W, et al. Adsorption mechanism at the molecular level between polymers and uremic octapeptide by the $2 \mathrm{D} 1 \mathrm{H}$ NMR technique. Biomacromolecules, 2006, 7: 1811-1818[DOI]

9 Tribet C, Porcar I, Bonnefont P A, et al. Association between hydrophobically modified polyanions and negatively charged bovine serum albumin. J Phys Chem B, 1998, 102(7): 1327-1333[DOI]

10 Wang B L, Wang J G, Ma Y, et al. Molecular docking and 3D-QSAR research of amidines of KARI inhibitor. Acta Chimica Sinica, 2006, 64(13): 1373-1378

11 胡建平, 常珊, 陈慰祖, 等. HIV-1 整合酶与抑制剂 LCA 的结合模式 及抗药性研究. 中国科学 B 辑: 化学, 2007, 37(3): 279-287 